\title{
Die Rolle der journalistischen Translation in der Übersetzerausbildung in Ungarn und einigen Nachbarländern
}

\author{
Viktor ZACHAR \\ Lehrstuhl für Übersetzen und Dolmetschen \\ Eötvös-Loránd-Universität Budapest, Philosophische Fakultät \\ zacharviktor@gmail.com
}

\begin{abstract}
Abstrakt. Die vorliegende Studie stellt die Ergebnisse einer mittels Fragebogen durchgeführten Umfrage über die Rolle der journalistischen Translation in der Übersetzerausbildung vor. Im Rahmen der Untersuchung antworteten insgesamt zweiundfünfzig, in der Übersetzerausbildung tätige Dozenten auf die formulierten Fragen. Ziel der Untersuchung war die Analyse dessen, welche Rolle die Übersetzung von Pressetexten in der Übersetzerausbildung an Hochschuleinrichtungen in Ungarn und in drei Nachbarländern (Kroatien, Rumänien und der Slowakei) spielt. Die Bedeutung des Fragebogens liegt darin, dass bislang keinerlei diesbezügliche Forschungen auf diesem Gebiet mit ungarischem Bezug veröffentlicht wurden und auch hinsichtlich der journalistischen Translation nur Forschungen existieren, die primär auf den Bereich Textlinguistik beschränkt sind.

Den obigen Tatsachen zum Trotz hat die durchgeführte Untersuchung die Bedeutung der Presseübersetzung im Übersetzungsunterricht aufgedeckt: Über 85 \% der Dozenten verwenden - aus unterschiedlicher Motivation heraus - Pressetexte, und fast $50 \%$ der Befragten setzen diese während der gesamten Dauer des Übersetzungsunterrichts ein. Die Fragen nach der Relevanz der journalistischen Translation bestätigen und begründen die Präsenz im Übersetzungsunterricht, da der Anteil derjenigen, die die Presseübersetzung aus Sicht der späteren Arbeit als Übersetzer für relevant halten, dank ihres breiten Anwendungsbereiches über 80 \% liegt.
\end{abstract}

Schlüsselwörter: journalistische Translation, Presseübersetzung, Übersetzungsunterricht

Abstract. The article presents a study on the role of journalistic translation (press translation) in translation teaching using a questionnaire in November-December 2016 on a sample of 52 translation teachers. The aim of the survey was to find out the role of journalistic translation in translation tea- 
ching in Hungary and three neighbouring countries: Croatia, Romania and Slovakia. The importance of the survey lies in the fact that no research has been published so far on this topic in Hungary, and only minor research has been done on the topic of journalistic translation itself (primarily limited to the field of text linguistics).

Despite these facts, the study has revealed the importance of journalistic translation in the teaching of translation: more than $85 \%$ of the teachers surveyed use press texts in teaching due to different motivations, and nearly $50 \%$ of respondents use them during the entire period of translation education. The inclusion of journalistic translation in the teaching of translation is also confirmed and justified by the fact that more than $80 \%$ of those surveyed consider the subject to be relevant for the students' future work as a translator due to the wide range of application areas of journalistic translation.

Keywords: journalistic translation, press translation, translation teaching

\section{Einleitung}

Die vorliegende Studie basiert auf einer mittels Fragebogen durchgeführten Umfrage unter Dozenten, ${ }^{1}$ die Übersetzungstechnik bzw. Fachübersetzen in Hochschuleinrichtungen in Ungarn bzw. einigen Nachbarländern unterrichten. Die Umfrage ist Teil eines größeren Forschungsprojekts, im Rahmen dessen ich die Situation eines Teilgebietes der Übersetzungsbranche, nämlich der journalistischen Translation (Presseübersetzung), in Ungarn beleuchte. Dabei möchte ich unter anderem ergründen, wer in Ungarn die in den Medien zweifelsohne stark präsente Übersetzung von Pressematerialien bzw. Nachrichten durchführt, inwieweit diese Fachleute qualifizierte Übersetzer sind, oder ob die Übersetzungstätigkeit in den Medien in Ungarn eher als eine Teilaufgabe der Journalisten angesehen wird.

Ziel dieser jetzigen Studie ist es zunächst zu untersuchen, welche Rolle die Übersetzung von Pressetexten in der Übersetzerausbildung in diversen Hochschulinstitutionen spielt. Dadurch kann einerseits ein bislang nur wenig erforschtes Gebiet der Ausbildung von Übersetzern besser kennengelernt, andererseits aber auch ein Beitrag zum oben genannten größeren Projekt geleistet werden.

\section{Journalistische Translation als Forschungsgegenstand}

Das verhältnismäßig große Thema der journalistischen Translation erweckte in den ersten Jahren des 21. Jahrhunderts das Interesse der Translationswissenschaft. Das stufenweise Vordringen und die allmähliche Verbreitung werden durch die Tatsache belegt, dass die zweite Auflage der Routledge Encyclopedia of Translation

1 Personenbezogene Ausdrücke in dieser Studie umfassen Frauen und Männer gleichermaßen. 
Studies (Baker-Saldanha 2009) aus dem Jahr 2009 sowie der erste Band des von Gambier und van Doorslaer herausgegebenen Handbook of Translation Studies (Gambier-van Doorslaer 2010) aus dem Jahr 2010 jeweils bereits einen Eintrag zum Thema enthalten. Quasi parallel zu diesen Erscheinungen widmeten renommierte translationswissenschaftliche Zeitschriften dem Thema Sonderausgaben: Im Jahr 2010 die Zeitschrift Across Languages and Cultures unter dem Titel „Translating Information in the Post-Industrial Society” (Jg. 11, Nr. 2), im Jahr 2012 META unter dem Titel ,Journalism and Translation” (Jg. 57, Nr. 4) sowie im Jahr 2015 die Zeitschrift Perspectives. Studies in Translatology unter dem Titel „Culture and News Translation” (Jg. 23, Nr. 4).

Wir können also im Einverständnis mit Valdeón (2015) feststellen, dass die Presseübersetzung in den vergangenen fünfzehn Jahren zu einem Forschungsgebiet der Translationswissenschaft geworden ist. Ihre Daseinsberechtigung wird - den Gedanken von Bani (2006) aufgreifend - unter anderem dadurch untermauert, dass sie zu einem viel breiteren Publikum Zugang findet, als zum Beispiel die literarische Übersetzung oder die Übersetzung von Theaterstücken. Ungeachtet dessen scheint das Thema in der ungarischen - aber wohlgemerkt auch in der deutschsprachigen - translationswissenschaftlichen Fachliteratur bislang nur marginal auf.

Diese Tatsache ist umso überraschender, als dass die Übersetzung von Pressetexten seit langen Jahren eine immens wichtige Rolle bei Sprachexamen, aber auch in der Übersetzerausbildung in Ungarn spielt, wie ich das aufgrund der Ergebnisse der Untersuchung im vierten Kapitel der vorliegenden Studie aufzeigen werde. Diese Rolle kann gleich zweifach begründet werden: Einerseits eignen sich Übungen der Presseübersetzung - vor allem zu Beginn der Ausbildung dazu, dass die Studierenden mit Hilfe von Pressetexten ihr Wissen bezüglich bestimmter Themen erweitern, die für die Fremdsprache charakteristischen Stile, Register, die übliche Wortwahl und das sprachliche Repertoire kennenlernen. Dies ist allein schon deshalb wichtig, weil aufgrund meiner Erfahrung die Studierenden vor Beginn ihrer Übersetzerausbildung kaum oder gar keine Tagesund Wochenzeitungen lesen.

Andererseits hat die Presseübersetzung einen Platz in der Ausbildung, weil während der späteren (freiberuflichen) Laufbahn - ebenfalls aufgrund meiner langjährigen Erfahrung - regelmäßig Situationen und Aufträge vorkommen, im Rahmen derer Pressetexte zu übersetzen sind. Diese können in zwei große Gruppen eingeteilt werden. In die erste Gruppe gehören die tatsächlich in Tages- oder Wochenzeitungen erscheinenden Artikel, deren Übersetzung deshalb in Auftrag gegeben wird, weil der Auftraggeber aus irgendeinem Grund Interesse am Inhalt des Artikels hat: So zum Beispiel, weil dieser ihr Unternehmen erwähnt oder behandelt, weil er die aktuelle politische, wirtschaftliche Lage analysiert, aktuelle Entwicklungen über die Branche oder Informationen über eine neue Regelung 
oder Norm enthält usw. In die andere Gruppe gehören Artikel, die in Mitarbeiterzeitschriften erscheinen und im Fall eines multinationalen Konzerns übersetzt werden müssen. Das Thema dieser Texte unterscheidet sich nicht bedeutend von denen der oben genannten Gruppe. Erwähnung finden neben diesen im Allgemeinen die Arbeitsbedingungen der Mitarbeiter, ihre Entlohnung, Entwicklungen und aktuelle Veranstaltungen im Unternehmen, Ausschreibungen sowie besondere Hobbys oder herausragende Ergebnisse bestimmter Mitarbeiter (Zachar 2013a; Zachar 2013b).

Trotz alledem könnte man die journalistische Translation als ein Forschungsgebiet der Translationswissenschaft bezeichnen, das lange Zeit hindurch auf unwürdige Weise vernachlässigt wurde und zum Teil immer noch wird. Aus dieser Sicht weist dieser Bereich ein ähnliches Schicksal auf wie die Erforschung des Übersetzens vom Blatt (Stegreifübersetzen), das bislang ebenfalls ein wenig erforschtes Gebiet der Translationswissenschaft ist (Felekné Csizmazia 2014). Nicht umsonst wird Letzteres von Parkin als ein Stiefkind der Translationswissenschaft

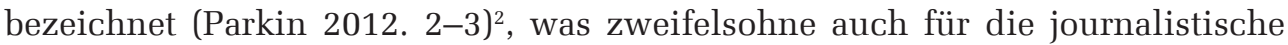
Translation gilt. Die Gründe dafür, dass die Presseübersetzung noch immer verhältnismäßig stiefmütterlich behandelt wird, lassen sich nicht eindeutig bestimmen. Vermutlich handelt es sich - darauf weisen auch die im späteren Verlauf dargelegten Ergebnisse der durchgeführten Studie hin - um ein Zusammenspiel mehrerer Faktoren. Der wohl wichtigste unter diesen könnte sein, dass aufgrund der journalistischen Praxis die Sprachmittlung in den Redaktionen - seit langen Jahrzehnten - nicht von professionellen Übersetzern versehen wird, bzw. dass diese Tätigkeit von den weiteren Redaktionsaufgaben und -prozessen, wie zum Beispiel dem eigenständigen Verfassen von Artikeln und dem Redigieren, nicht unterschieden wird (Bielsa-Bassnett 2009. 57). Gerade deshalb hatten lange Zeit hindurch weder die Journalisten noch die Übersetzungsbranche (einschließlich der Translationswissenschaft) das Gefühl, dass dieses Gebiet einer näheren Untersuchung würdig wäre - obwohl es aus translationswissenschaftlicher Sicht als vollkommen legitim bezeichnet werden kann, den Übersetzungsprozess als Adaptation und Selektion zu betrachten (vgl. z. B. Hu 2003). Die Einbettung der Übersetzungstätigkeit in die anderen journalistischen Prozesse wird auch durch die (textlinguistische) Tatsache bekräftigt, dass es aufgrund der Vielzahl an Quellen, auf denen ein Zeitungsartikel basiert, eine schier unmögliche Aufgabe darstellt, eine Nachricht in ihre Komponenten aufzugliedern, um zu bestimmen, welche Komponenten das Ergebnis des Redigierens und welche das Ergebnis von Übersetzungsprozessen darstellen (van Doorslaer 2009. 85).

2 Für den Hinweis auf diesen Vergleich bezüglich des Stehgreifübersetzens bin ich meiner Kollegin Erzsébet Felekné Csizmazia von der Eötvös-Loránd-Universität Budapest zum Dank verpflichtet. 
Eine mit den obigen Ausführungen gewissermaßen zusammenhängende Erklärung könnte auch sein, dass die journalistische Translation (in den Redaktionen) als eine Art natürliche Übersetzungssituation (Harris-Sherwood 1978) angesehen wird - in Unterscheidung zu den professionellen Übersetzungen und Übersetzungssituationen. Aus diesem Grund wurde sie - zumindest lange Zeit hindurch - als der Erforschung unwürdig angesehen.

Ein weiterer möglicher Grund - der vor allem aus Sicht der Übersetzerausbildung (aber auch der Übersetzerpraxis) als Erklärung dienen könnte - ist, dass Pressetexte nur schwer kategorisierbar sind und weder eindeutig dem Bereich der literarischen noch der Fachübersetzung zugeordnet werden können. In der Übersetzerausbildung in Ungarn - wie auch in der Praxis - können grundsätzlich jedoch gerade diese beiden großen Bereiche unterschieden werden. Aus diesem Aspekt verweise ich auf die bereits erwähnte Parallele zum Übersetzen vom Blatt (Stegreifübersetzen), das ebenfalls weder eindeutig dem Bereich des Übersetzens noch des Dolmetschens zugeordnet werden kann. Deshalb spielt diese Tätigkeit in der Ausbildung in Ungarn keine allzu relevante Rolle und wird auch nicht gesondert, im Rahmen eines eigenen Kurses unterrichtet. Mit dieser Tatsache hängt auch die Feststellung zusammen, dass die Pressübersetzung aufgrund der Vielzahl an Textsorten nur schwer als eine Einheit aufgefasst werden kann. Róka zum Beispiel unterscheidet dreiundzwanzig Textsorten innerhalb des Journalismus, vom Leitartikel über die Meldung (Nachricht) - die sie in insgesamt acht Untergruppen gliedert - bis hin zur Glosse und zum Kommentar (Róka 1986. 204).

Schließlich könnte aus Sicht der Übersetzungsbranche als Erklärung dienen, dass die journalistische Translation hinsichtlich der Zahl der Aufträge weit weniger ins Gewicht fällt als die Fachübersetzung. Zwar kommen Situationen, in denen eine Presseübersetzung notwendig ist, - wie bereits früher erwähnt - regelmäßig vor, jedoch bleibt die Zahl dieser weit hinter der Zahl der Fachübersetzungsaufgaben zurück. Diese mögliche Erklärung ist aufgrund der weiter unten vorgestellten Antworten auf die entsprechenden Fragen der durchgeführten Studie von besonderer Wichtigkeit. In diesem Zusammenhang müssen wir wohlgemerkt in erster Linie an die Arbeit als freiberuflicher Übersetzer oder als Übersetzer in einem Übersetzungsbüro denken, weniger an die Arbeit in einer Redaktion oder bei einer Nachrichtenagentur, zumal Letztere, wie weiter oben genannt, aufgrund der internationalen Forschungen charakteristischer Weise nicht von professionellen Übersetzern versehen wird.

\section{Vorstellung der Untersuchung}

Zwischen dem 21. November und dem 5. Dezember 2016 führte ich mit Hilfe eines Online-Fragebogens eine Studie unter Dozenten in Einrichtungen der Übersetzerausbildung durch. Dabei zog ich nicht nur Institutionen in Ungarn, 
sondern auch solche in einigen umliegenden Ländern in Betracht, um mir einen größeren Überblick zu verschaffen. Ziel der Untersuchung war es zu ermitteln, welche Rolle die Übersetzung von Pressetexten in der Übersetzerausbildung in diversen Hochschulinstitutionen spielt. Dabei wurde einerseits der Dolmetschunterricht - der sowohl in Ungarn als auch in einigen umliegenden Ländern zumindest teilweise parallel zum Übersetzungsunterricht läuft - bewusst aus der Untersuchung ausgeklammert, obwohl ich mir darüber im Klaren bin, dass sich Pressetexte hervorragend zum Übersetzen vom Blatt (Stegreifübersetzen), zum Verfassen von zu verdolmetschenden Texten für die Studierenden oder zur Vorbereitung auf ganz konkrete Dolmetschaufgaben bzw. Dolmetschaufträge eignen. Andererseits muss an dieser Stelle erwähnt werden, dass die Übersetzung von Pressetexten in der hier behandelten Übersetzerausbildung im Grunde ausschließlich das (meist vollständige) Eins-zu-eins-Übersetzen von Texten bedeutet und mit der Arbeit mit Texten in Redaktionen oder Nachrichtenagenturen nicht wirklich vergleichbar ist. Die weitere Erörterung dieses Punktes wird ebenfalls eine Aufgabe des eingangs erwähnten größeren Forschungsprojekts sein.

Da es sich um einen in breitem Kreis zugänglichen und frei teilbaren Online-Fragebogen handelte, ist die genaue Zahl derjenigen, die den Fragebogen erhalten haben, nicht ermittelbar. Insgesamt wurden aber zweiundfünfzig ausgefüllte Fragebögen zurückgeschickt: zwölf davon von Hochschuleinrichtungen in den erwähnten Nachbarländern (Kroatien, Rumänien und der Slowakei) und vierzig von Dozenten, die an ungarischen Hochschuleinrichtungen lehren. Somit waren insgesamt achtzehn verschiedene Institutionen vertreten, aus Ungarn vierzehn und aus den drei erwähnten Ländern vier Hochschulen bzw. Universitäten.

Der Fragebogen bestand aus zwölf Fragen sowie einer Möglichkeit für Anmerkungen am Ende, wobei die letzten beiden Fragen und die Anmerkungen die Möglichkeit für Freitextantworten boten. Die restlichen zehn Fragen beinhalteten jeweils vorgegebene Antwortmöglichkeiten. In den ersten beiden Fragen ermittelte ich die Institution der den Fragebogen ausfüllenden Dozenten und die unterrichtete Fremdsprache. Die dritte Frage bezog sich darauf, ob die Befragten zurzeit im Rahmen des Übersetzungs- oder Übersetzungstechnik-Seminars Pressetexte übersetzen lassen, oder falls nicht - so die vierte Frage -, ob sie früher solche Texte im Unterricht verwendet haben. Die fünfte Frage suchte - mit Hilfe von vorgegebenen Antwortmöglichkeiten - nach einer Antwort darauf, was der Grund dafür ist, dass die Befragten keine solchen Texte übersetzen lassen bzw. ließen. Die sechste Frage erkundigte sich nach der Motivation für das Übersetzen von Pressetexten, ebenfalls mit vorgegebenen Antwortmöglichkeiten. Bei der siebten Frage hatten die Befragten die Möglichkeit anzugeben, wann sie während der gesamten Dauer der Übersetzerausbildung Pressetexte verwenden: zu Beginn, gegen Mitte, am Ende der Ausbildung oder durchweg während der gesamten Ausbildung. Die achte Frage erkundigte sich mittels vorgegebener Antwort- 
möglichkeiten nach den angewandten Textsorten innerhalb der Presseübersetzung, die neunte nach dem genauen Thema dieser Texte. Die zehnte Frage wollte wissen, ob die Presseübersetzung den Befragten zufolge aus Sicht der späteren Übersetzungsarbeit relevant sei oder nicht. Falls ja, so die elfte Frage, wo können solche Situationen im späteren Verlauf vorkommen, falls nicht (zwölfte Frage), was ist der Grund dafür?

\section{Bewertung der Ergebnisse der Untersuchung}

\subsection{Bewertung der Antworten auf die ersten fünf Fragen}

Wie bereits erwähnt, stammen die Antworten des Fragebogens von zwölf Dozenten an Hochschuleinrichtungen in den umliegenden Ländern (Kroatien, Rumänien und der Slowakei) und von vierzig Dozenten, die an Hochschuleinrichtungen in Ungarn lehren. Aus Ungarn waren vierzehn verschiedene Institutionen vertreten: Eötvös-Loránd-Universität Budapest (10 Dozenten), Technische Universität Budapest (6), Kodolányi-János-Hochschule Székesfehérvár (5), Universität Szeged (4), Károli-Gáspár-Universität der Reformierten Kirche Budapest (3), Eszterházy-Károly-Universität Eger (3), Szent-István-Universität Gödölló (2), Wirtschaftsuniversität Budapest (1), Universität Óbuda in Budapest (1), Pázmány-Péter-Universität der Katholischen Kirche Budapest (1), Universität Miskolc (1), Universität Nyíregyháza (1), Pannonische Universität Veszprém (1) und Universität Pécs (1). Vier Einrichtungen befinden sich dagegen in den drei oben erwähnten Nachbarländern Ungarns: Sapientia Universität Klausenburg (6 Dozenten), Konstantin der Philosoph-Universität Nitra (3), Josip-Juraj-Strossmayer Universität Osijek (2) und Universität Bukarest (1).

Die obigen Antworten auf diese erste Frage nach der Hochschuleinrichtung zeigen, an wie vielen Hochschulen bzw. Universitäten Ungarns überhaupt Übersetzen gelehrt wird. Dabei ist der Vollständigkeit halber anzumerken, dass die Ausbildung nicht in jedem Fall in einem Masterstudiengang erfolgt, sondern zum Teil in Form einer fachspezifischen Weiterbildung, wobei Ersterer im Allgemeinen zwei Jahre, letztere Weiterbildung ein Jahr dauert. Dies ist aus Sicht der weiteren Fragen bzw. der durchgeführten Studie nur insofern von Bedeutung, als dass im Rahmen eines zweijährigen Studiums mehr Übersetzungskurse angeboten werden (bzw. absolviert werden müssen) und somit - zumindest theoretisch - auch mehr Möglichkeiten zum spezifischen Unterricht von Presseübersetzung gegeben sind.

Bei der Frage nach der unterrichteten Fremdsprache - wobei Mehrfachnennungen möglich waren -, belegte, wie zu erwarten war, Englisch mit 28 Antwor- 
ten den ersten Platz (53,8 \%) vor Deutsch (15 Antworten, 28,8 \%). Weit abgeschlagen folgen Französisch und Rumänisch (jeweils 4 Antworten), Italienisch (3), Spanisch, Slowakisch und Kroatisch (2) sowie Niederländisch, Russisch, Chinesisch, Japanisch, Finnisch und Ungarisch (1).

Bei der dritten Frage, ob nämlich die Dozenten zurzeit im Rahmen des von ihnen geleiteten Übersetzungs- oder Übersetzungstechnik-Seminars Pressetexte übersetzen lassen, antwortete die überwiegende Mehrheit mit ,ja': 45 von 52 Befragten (86,5 \%) gaben an, im Übersetzungsunterricht zurzeit mit solchen Texten zu arbeiten und lediglich 7 Personen (13,5 \%) verzichten im Unterricht auf Pressetexte. Aber auch diese letztere Gruppe arbeitete zumindest früher im Übersetzungsunterricht mit solchen Texten: Auf die vierte Frage nämlich, ob die Befragten früher solche Texte im Unterricht verwendet haben, auch wenn sie momentan keine Pressetexte einsetzen, antworteten alle ausnahmslos mit ,ja‘. Die Zahl der Antworten bei dieser Frage (17) weist darauf hin, dass ein Teil der Befragten die Frage missverstanden hat und auch auf diese Frage geantwortet hat, obwohl sie zurzeit mit solchen Texten arbeiten. Das beeinflusst aber keineswegs das mehr als eindeutige Ergebnis von $100 \%$.

Der Grund dafür, weshalb die betroffenen Dozenten zurzeit keine Pressetexte einsetzen, auch wenn sie früher mit solchen Texten gearbeitet haben (fünfte Frage), konnte dagegen nicht zufriedenstellend ermittelt werden, weil lediglich eine einzige Person von den potentiellen sieben, die die dritte Frage mit ,nein beantwortet hatten, eine Antwort gab. Dieser Befragte führte von den vorgegebenen Antworten mit der Möglichkeit einer Mehrfachnennung gleich zwei Gründe an: Einerseits, dass es sich nicht ausgesprochen um eine Aufgabe für literarische Übersetzer bzw. Fachübersetzer handle, und andererseits dass Pressetexte aus der Sicht der späteren Arbeit der Sprachmittler nicht relevant seien. Aufgrund dieser einzigen Antwort können wir im Fall der 13,5 \% der Befragten, die zurzeit nicht mit Pressetexten arbeiten, auch weiterhin nur vermuten, welche Erklärung dahintersteckt. Wie auch in den vorgegebenen Antwortmöglichkeiten angeführt, vermute ich dahinter - über die beiden obigen Nennungen hinaus - folgende mögliche Gründe: Für die Übersetzung solcher Texte bleibt im Rahmen der straffen Ausbildung kein Platz; im Vergleich zu literarischen Texten bzw. Fachtexten werden sie als zu einfach eingestuft und deshalb ausgelassen; die Dozenten sind der Ansicht, dass solche Texte die Fach- oder die Sprachkenntnisse der Studenten nicht entsprechend fördern und schließlich, dass Letztere nur ungern solche Texte übersetzen bzw. sie für langweilig halten.

Aufgrund der obigen Fragen und der auf diese gegebenen Antworten ist ersichtlich, dass Pressetexte in der Übersetzerausbildung in Ungarn und auch in einigen Einrichtungen der umliegenden Länder von fundamentaler Bedeutung sind. Dies wird zudem durch zwei weitere Fakten untermauert: Einerseits durch die Tatsache, dass zum Beispiel an der Eötvös-Loránd-Universität Budapest bei 
den Aufnahmeprüfungen im Allgemeinen Pressetexte bei der Übersetzungsaufgabe übersetzt werden müssen. Andererseits dadurch, dass bei den Übersetzerprüfungen für werdende Sprachmittler, die ihr Wissen ohne eine gesonderte Übersetzerausbildung auf die Probe stellen wollen, an derselben Universität ebenfalls zumeist Pressetexte eingesetzt werden. Mögliche Gründe dafür sind vermutlich - um ein wenig bereits auf die nächste Frage vorzugreifen -, dass es sich um öffentliche, leicht zugängliche Texte handelt, dass sie stets aktuell sind und sich somit auf dem neuesten Stand befinden und drittens, dass sie keiner Geheimhaltungserklärung unterliegen, was bei Fachübersetzungen verständlicher Weise sehr oft der Fall ist. ${ }^{3}$ Schließlich sollten an dieser Stelle noch zwei weitere Gründe erwähnt werden. Einerseits, dass Pressetexte im Unterricht im Allgemeinen nicht als Fach-, sondern als „allgemeine“ Texte eingestuft werden, weil sie nicht Texte sind, die zwingend von einem Fachmann eines bestimmten Fachbereichs für ein Fachpublikum verfasst wurden und (deshalb) charakteristischer Weise nicht viele Termini enthalten. Andererseits, dass sich Pressetexte - aufgrund meiner Erfahrung, aber auch aufgrund der Antworten auf die weiteren Fragen des Fragebogens - hervorragend zur Aneignung, Einübung und Messung der Fähigkeiten, Fertigkeiten und des Wissens im Bereich Übersetzen und Übersetzungstechniken eignen.

Die obigen Feststellungen bestätigen zudem die international zu beobachtenden und weiter oben auch zitierten Tendenzen in der Translationswissenschaft, wonach die journalistische Translation durchaus einen legitimen, wenn auch recht neuen Forschungsgegenstand dieser Wissenschaft darstellt, allein schon deshalb, weil sie - zumindest in Ungarn, aber vermutlich in etlichen anderen Ländern genauso - sehr stark in der Übersetzerausbildung präsent ist. Zugleich werfen die oben zitierten Ergebnisse aber auch Fragen auf, vor allen Dingen, warum sich die Translationswissenschaft erst verhältnismäßig spät, in den 2000er-Jahren mit diesem Phänomen zu beschäftigen begann, bzw. warum zum Beispiel in Ungarn bis heute keine diesbezüglichen Forschungen durchgeführt wurden. An dieser Stelle muss erwähnt werden, dass die journalistische Translation in der ungarischsprachigen translationswissenschaftlichen Fachliteratur lediglich in Bezug auf einen engen Teilbereich bzw. einen bestimmten Aspekt erscheint. Das Aufzeigen und die Erforschung weiterer Aspekte sind bislang nicht erfolgt. Bei diesem Teilbereich handelt es sich innerhalb der Translationswissenschaft um die Textlinguistik, wobei diesbezüglich die Forschungen und Publikationen von Károly und Bánhegyi zu erwähnen sind. Während sich Erstere in erster Linie im Zusammenhang mit der Erforschung der Textsorten (z. B. Károly 2007), der Kohäsionsmittel (z. B. Károly et al. 2012) und der rhetorischen Struktur (z. B. Károly 2013) mit dem Thema auseinandersetzt, konzentriert sich Letzterer an-

3 Für den Hinweis auf diese Gründe bin ich meiner Kollegin Edina Robin von der Eötvös-LorándUniversität Budapest zum Dank verpflichtet. 
hand von Zeitungstexten primär auf den Aspekt der politischen Diskursanalyse (z. B. Bánhegyi 2012) und die Fragen der Manipulation durch die politische Kommunikation (z. B. Bánhegyi 2013). Bestätigt wird diese Tatsache im Übrigen auch durch Valdeón (2015), der in seiner Studie, in der er einen Überblick über die bisherige Geschichte der journalistischen Translation gibt, in Bezug auf die ungarischen Forschungen lediglich auf die erwähnten Arbeiten von Károly im Bereich Textlinguistik verweist.

\subsection{Bewertung der Antworten auf die nächsten vier Fragen}

Die sechste Frage des Fragebogens bezog sich darauf, mit welchem Ziel die Dozenten Pressetexte übersetzen lassen, was also ihre diesbezügliche Motivation ist. Bemerkenswerter Weise liegt die Mehrheit der Antworten ziemlich knapp beieinander. Von den vorgegebenen Antwortmöglichkeiten (Mehrfachnennungen waren auch in diesem Fall möglich) wurde mit insgesamt 40 Antworten (76,9 \%) am häufigsten genannt, dass sie den Studenten die verschiedenen Gattungen/ Textsorten vorstellen möchten. Knapp dahinter an zweiter Stelle steht - damit gewissermaßen im Zusammenhang - der Wunsch nach Erweiterung verschiedener Stile bzw. sprachlicher Register bei ihnen (36 Nennungen, 69,2 \%). Nur einmal weniger wurde die Erweiterung der übersetzungstechnischen Kenntnisse als Ziel genannt, wozu sich aufgrund der Antworten Pressetexte ebenfalls hervorragend eignen (35 Nennungen, 67,3 \%). An vierter Stelle liegt die Erweiterung des Wortschatzes (30 Nennungen, 57,7 \%), gefolgt von der Vorbereitung auf die spätere Arbeit als Übersetzer, wo solche Texte ebenfalls durchaus vorkommen können (27 Nennungen, 51,9 \%). Insgesamt 26 Mal wurde die Erhöhung der übersetzerischen Kreativität (50 \%) und $22 \mathrm{Mal}$ die Erweiterung des Wissens über die Welt genannt (42,3\%).

Unter den sonstigen, nicht mehr vorgegebenen Antworten finden sich die folgenden sieben: Ein Befragter nannte an dieser Stelle im Grunde übersetzungstechnische Beispiele, nämlich die richtige Schreibweise von Zahlen, die richtige Verwendung des Dezimaltrennzeichens (Dezimalpunkt oder Dezimalkomma), die Vorgehensweise bei fehlerhaften zielsprachlichen Sätzen und die Übersetzung von Realien (kulturspezifischen Termini). Ein weiterer Befragter erwähnte an dieser Stelle die Weitergabe von kontrastiven grammatischen und lexikalischen Kenntnissen. Eine dritte Antwort wies auf die Aneignung einer fließenden, den zielsprachlichen Normen entsprechenden Formulierungsweise und die Gewöhnung an das Lesen der tagesaktuellen Nachrichten hin, wobei Letzteres auch ein zweites Mal ergänzend erwähnt wurde. Auf diese Problematik habe ich selbst bereits im zweiten Kapitel meiner vorliegenden Arbeit kurz hingewiesen. Ebenfalls genannt wurden an dieser Stelle: das Üben der Übersetzung von Schlagzeilen, die Verwendung von Pressetexten im Dolmetschunterricht zum Übersetzen 
vom Blatt (Stegreifübersetzen), das Aufzeigen des Sprachwandels und schließlich das Übersetzen von Kurznachrichten, die aufgrund ihrer Einfachheit und Kürze auch bei unerfahrenen Studenten eingesetzt und im Rahmen einer Seminarstunde komplett besprochen werden können.

Die Tatsache, dass die Mehrheit der Antworten eng beieinander liegt und auch die - mit Ausnahme der sonstigen - am seltensten angekreuzte Antwortmöglichkeit mit einer Häufigkeit von 42,3 \% vorkam, weist zweifelsohne darauf hin, dass der Einsatz von Pressetexten im Übersetzungsunterricht aus einer Vielzahl von Gründen, mit unterschiedlicher Motivation erfolgt und nicht ein einziger wichtiger, über den anderen stehender Grund auszumachen ist. Obwohl das Übersetzen von Zeitungsartikeln in der Ausbildung aufgrund meiner Erfahrung im Kreis der Auftraggeber (am ehesten der Übersetzungsbüros) keinen guten Ruf zu haben scheint, deuten die obigen Antworten eindeutig auf eines hin: Aufgrund der Meinung der das Übersetzen lehrenden Personen eignen sie sich aus didaktischem Aspekt, aus den unterschiedlichsten Gründen hervorragend für den Übersetzungsunterricht.

Dieser Gedanke wird auch durch die auf die siebte Frage gegebenen Antworten bestätigt. Die Befragten konnten hier anführen (Mehrfachnennungen waren möglich), wann sie während der gesamten Dauer der Übersetzerausbildung diese Pressetexte einsetzen: zu Beginn, gegen Mitte, am Ende der Ausbildung oder durchweg während der gesamten Ausbildung. Vor der Durchführung der Umfrage hatte ich vermutet, dass bei dieser Frage ganz eindeutig die erste Antwort am häufigsten genannt werden würde, und zwar gleich aus zwei Gründen: Einerseits, weil Zeitungsartikel im Vergleich zu Fachtexten, aber auch zu literarischen Texten in der Mehrzahl der Fälle einfacher zu übersetzen sind, das heißt im Allgemeinen weniger Forschungsarbeit benötigen und auch weniger terminologische Probleme aufwerfen. Andererseits, weil das Übersetzen von Pressetexten im Vergleich zu Fachtexten viel seltener vorkommt und deshalb andere Texte während des Übersetzungsunterrichts zu einem größeren Anteil eingeplant und geübt werden müssten. Das ist aufgrund meiner Erfahrung auch einer der am häufigsten genannten Gründe, warum Pressetexte von den Übersetzungsbüros als potentiellen zukünftigen Auftraggebern nicht so gerne in der Ausbildung gesehen werden.

Demgegenüber lässt die Mehrheit der Dozenten, nämlich 24 Personen (47,1 \%), während der gesamten Ausbildung Pressetexte übersetzen, was einen überraschend hohen Anteil darstellt. Es folgen der Beginn der Ausbildung (15 Antworten, 29,4 \%) und die Mitte der Ausbildung (13 Antworten, 25,5 \%), wogegen nur 2 Personen (3,9 \%) ankreuzten, Zeitungsartikel erst gegen Ende der Ausbildung einzusetzen. Auch wenn wir also die übrigen Antworten im Vergleich zur gesamten Dauer zusammenrechnen, verwenden fast genauso viele Lehrkräfte durchweg Pressetexte, wie solche, die diese nur streckenweise einsetzen. Dies ist aufgrund 
meiner oben genannten, vorab formulierten Vermutungen auf jeden Fall erstaunlich, und stärkt erneut die Feststellung, dass die Presseübersetzung im Übersetzungsunterricht und in der Wissenschaft eine tatsächliche Daseinsberechtigung hat. Wobei an dieser Stelle natürlich auch erwähnt werden muss, dass diese Zahlen umgekehrt selbst für den verhältnismäßig schlechten Ruf der Presseübersetzung verantwortlich sein können: Vor allem im Fall eines durchgängigen Einsatzes müssen dadurch andere Textsorten notgedrungen zu kurz kommen, die ihrerseits viel häufiger in der beruflichen Praxis der Übersetzer vorkommen.

Im Rahmen der achten Frage wollte ich wissen, welche Textsorten innerhalb der Übersetzung von Pressetexten von den Dozenten im Rahmen des Übersetzungsunterrichts bevorzugt werden (Mehrfachnennungen waren erneut möglich). Von den vorgegebenen Antwortmöglichkeiten wurden ausnahmslos alle genannt. An erster Stelle liegen mit 37 Antworten (71,2 \%) (analysierende) Zeitungsartikel, mit einigem Abstand folgen Kurznachrichten (24 Antworten, 46,2 \%), Reportagen (22 Antworten, 42,3 \%) und Leitartikel (19 Antworten, 36,5 \%). An fünfter Stelle stehen Mitteilungen (15 Antworten, 28,8 \%), an sechster Stelle Interviews (11 Antworten, 21,2 \%), auf dem siebten Platz Kritiken (10 Antworten, 19,2 \%) und auf dem achten Platz Kommentare/Glossen (9 Antworten, 17,3 \%). Am seltensten genannt wurden Rezensionen (7 Antworten, 13,5 \%), Leserbriefe (5 Antworten, 9,6 \%) und drei weitere Textsorten unter Sonstiges (die jedoch ohne weiteres in die vorgegebenen Kategorien eingeordnet werden könnten): wissenschaftliche Fachartikel, populärwissenschaftliche Artikel sowie wirtschaftliche Kurznachrichten bzw. Kurzberichte.

Die Struktur der Antworten ist in diesem Fall nicht überraschend und folgt meines Erachtens der in der Praxis anzutreffenden Häufigkeit. Dies trifft sowohl aus Sicht der Zahl der authentischen quellsprachlichen Texte als auch aus Sicht der bei den Übersetzungsaufträgen gefragten Textsorten innerhalb der Übersetzung von Pressetexten zu. Anzumerken ist, dass Kurznachrichten vermutlich aufgrund ihrer Kompaktheit und Kürze im Unterricht bevorzugt werden, wobei aber auch die ausführlichere Textsorte der Reportage fast mit identischer Häufigkeit unter den genannten Antworten vorkommt. Die Tatsache, dass alle vorgegebenen Antwortmöglichkeiten auch tatsächlich genannt wurden bestätigt wiederum das Ergebnis der sechsten Frage: Dort wurde am häufigsten als Ziel erwähnt, dass die Dozenten den Studenten die verschiedenen Gattungen/Textsorten näherbringen möchten.

Ziel der neunten Frage war es, das Thema bzw. die Themen der im Unterricht verwendeten Pressetexte näher zu beleuchten. Aufgrund der vorgegebenen Antwortmöglichkeiten - mit der Möglichkeit der Mehrfachnennung - wurde am häufigsten das Thema Wirtschaft/Finanzen genannt (41 Antworten, 78,8 \%), gefolgt von Politik (34 Antworten, 65,4 \%) und dem öffentlichen Leben (33 Antworten, $63,5 \%)$. Weit abgeschlagen dagegen die weiteren Antwortmöglichkeiten: Na- 
turwissenschaften (18 Antworten, 34,6 \%), Technik (5 Antworten, 9,6\%), Stars und Sternchen (4 Antworten, 7,7 \%) sowie Sport (2 Antworten, 3,8 \%). Unter Sonstiges findet sich zudem der Bereich Kultur (2 Nennungen) sowie folgende, jeweils nur einmal genannte Themen: Europäische Union, Verkehr, Bildungswesen, Recht, Geschichte, Linguistik und Tourismus.

Das Ergebnis ist auch in diesem Fall nicht überraschend: Der Bereich Wirtschaft/Finanzen gehört zu den wichtigsten Fachgebieten späterer Übersetzer. Auch im Rahmen der Übersetzerausbildung an der Philosophischen Fakultät der Eötvös-Loránd-Universität Budapest liegt einer der Schwerpunkte auf der Übersetzung solcher Fachtexte (der andere Schwerpunkt ist Recht). Die Übersetzung von Pressetexten im Themenbereich Politik und öffentliches Leben entspricht wiederum der Struktur der Antworten bei Frage sechs (Ziel der Presseübersetzung): Diese eignen sich nämlich hervorragend zur Erweiterung verschiedener Stile bzw. sprachlicher Register, zur Erweiterung der übersetzungstechnischen Kenntnisse, des Wortschatzes und natürlich des Wissens über die Welt.

\subsection{Bewertung der Antworten auf die letzten vier Fragen}

Drei der letzten vier Fragen bezogen sich auf die Relevanz der Presseübersetzung im Rahmen der späteren übersetzerischen Arbeit. Auf die zehnte Frage, ob die Übersetzung solcher Texte aus dieser Sicht relevant sei, antworteten 43 Befragte mit ,ja', das sind 82,7 \%, und lediglich 17,3 \%, also 9 Personen, mit ,nein'. Damit fällt das Ergebnis bei dieser Frage weit deutlicher aus, als ich vorab erwartet habe. Auch wenn die Zahlen sich nicht vollkommen decken, ist hier durchaus auch eine starke Korrelation zu den Ergebnissen bei der dritten Frage feststellbar, wo 86,5 \% geantwortet haben, derzeit im Übersetzungsunterricht mit solchen Texten zu arbeiten. Das bedeutet, dass die überwiegende Mehrheit der Befragten der Ansicht ist: Die Übersetzung solcher Texte ist auch aus beruflicher Sicht relevant und dient nicht nur der Vermittlung übersetzungstechnischer Kenntnisse, von Fachwissen usw.

Diese Feststellung wird durch die Freitextantworten auf die elfte Frage bestätigt: Im Fall einer bejahenden Antwort auf die vorangehende Frage richtete sich diese darauf, wo, in welchen Situationen Presseübersetzung während der Übersetzerarbeit vorkommt bzw. vorkommen kann. Insgesamt 41 Personen antworteten mehr oder weniger ausführlich auf diese Frage, das heißt, mit Ausnahme zweier Personen alle, die auf die zehnte Frage mit ,ja' geantwortet haben. Die diesbezüglichen Rückmeldungen können grob in zehn Kategorien eingeteilt werden. In die erste gehören die allgemeinen Hinweise auf die übersetzerische Arbeit (z. B. „man kann nicht vorhersagen, was das Leben bringen wird, deshalb setze ich bei der Zusammenstellung der Texte auf Vielfältigkeit“), auf übersetzungstechnische Fragen (z. B. „Pressetexte verwenden eine charakteristische Sprache, die gut bei 
anderen Textsorten genutzt werden kann“; „die hier gewonnenen Kenntnisse können auch bei der Fachübersetzung genutzt werden“; „Übersetzungsstrategien, Stile, Register können damit hervorragend geübt werden“).

In die zweite Kategorie gehören die Antworten, die auf die Pressebeobachtung und Presseschau hinweisen (z. B. ,auch in der Form, wo es nicht um klassische Übersetzung, sondern Verdichtung geht /weil ein Teil unserer Studenten nicht als klassischer Übersetzer angestellt wird, sondern als Teil des Arbeitsbereichs, zum Beispiel als Ökonom oder Ingenieur, übersetzt, ist auch diese Aufgabe relevant/“; „Erstellen von Zusammenfassungen aus einem oder mehreren Artikeln, zum Beispiel an Botschaften oder in internationalen Organisationen"). Damit im Zusammenhang steht die dritte Gruppe von Antworten: das Erscheinen der Übersetzung von Pressetexten in der Arbeit von Nachrichtendiensten, Nachrichtenagenturen (z. B. „bei der Mehrheit der von Nachrichtenagenturen übernommenen Nachrichten handelt es sich um Übersetzungen“; „diese erhalten regelmäßig derartige Übersetzungsaufträge, aber meine Erfahrung ist, dass hier Personen übersetzen, die keinen Abschluss als Übersetzer haben“). Die nähere Untersuchung dieser letzteren Anmerkung ist meines Erachtens auf jeden Fall wichtig, denn aufgrund der internationalen Fachliteratur ist sie außerhalb Ungarns stichhaltig. Die Überprüfung im ungarischen Kontext wird Aufgabe des eingangs erwähnten, zurzeit laufenden größeren Forschungsprojekts sein.

Die vierte Antwortgruppe beinhaltet den Hinweis auf die Anwendung der Presseübersetzung im Fall von offiziellen Mitteilungen, Presseerklärungen und ähnlichen (z. B. „Kommunikationstätigkeit von Filmemachern, Künstlern und Verlagen“; „Regierungskommunikation“; „Presseabteilung von Ämtern“; „Arbeit an diplomatischen Vertretungen"). Damit im Zusammenhang steht die fünfte und sechste Gruppe: Einerseits die Übersetzung von publizistischen Texten für Firmen und als Übersetzer/Assistent bei multinationalen Unternehmen (z. B. „im Fall von Zweisprachigkeit bzw. einer Minderheitensprache sind Kenntnisse im Bereich Presseübersetzung im Fall mehrerer Arbeitsbereiche notwendig“; „Übersetzen von Artikeln über neue technische Entwicklungen von Firmen“). Andererseits das Erstellen der fremdsprachigen Version von Internet-Nachrichtenportalen bzw. das Übersetzen von Webseiten sowie von Pressenachrichten für solche.

Die siebte Gruppe von Antworten bezog sich auf die Übersetzung von kreativen Texten sowie solchen im Bereich Marketing (z. B. „manchmal muss man gleichzeitig Werbetexter sein“; ,,im Fall von Werbekampagnen bestimmter Produkte können solche Texte vorkommen“). Dagegen wurde im Fall der achten Gruppe die Zusammenstellung und Beurteilung von Bewerbungen als Anwendungsgebiet erwähnt, das nirgendwo eingeordnet werden kann und deshalb eine eigenständige Gruppe bildet. In die vorletzte Gruppe gehört die Erwähnung des Kontextes der Europäischen Union (z. B. „in diesem Fall kann sie auch Teil von 
Arbeitsbereichen sein /nicht nur von Übersetzern, zum Beispiel in der Verwaltung/“; „Im Fall der juristischen Fachübersetzung ist die detaillierte und eingehende Kenntnis der nationalen, internationalen und EU-Terminologie wichtig. In Pressetexten kommen diese auf eigentümliche Weise gleichzeitig vor, wobei diese Texte die zu den jeweiligen Registern/zur nationalen Terminologie gehörenden Termini eng voneinander abgrenzen können und so den Übersetzern einen guten Überblick über die diesbezüglichen Unterschiede verschaffen“).

In die zehnte und letzte Gruppe von Antworten gehört schließlich die Erwähnung der Übersetzung von Artikeln für Zeitungen und Zeitschriften, wobei konkret die Zeitschriften Heti Válasz, HVG (Übersetzen von Artikeln der Zeitschrift The Economist), National Geographic und Geo erwähnt wurden (z. B. „Übersetzen von Meinungen/Studien ausländischer Verfasser; „Studien ausländischer Verfasser in wissenschaftlichen Zeitschriften“; Übersetzen von Interviews für Nachrichtenportale oder sonstige Seiten“). Wichtig ist, dass in dieser Kategorie auch erwähnt wurde: Die Nachrichten werden oftmals nicht eins zu eins übersetzt, sondern überarbeitet (,„̈̈berarbeitung von /naturwissenschaftlichen/ Nachrichten in einer anderen Sprache für Leser mit abweichenden Vorkenntnissen bzw. abweichendem Wissensstand /z. B. Fachleute, Mittelschüler, Kinder/. Dabei handelt es sich in erster Linie nicht um eine Übersetzungsaufgabe, sondern um die Angleichung des Inhalts des übersetzten Materials an das Niveau der Lesergruppen.").

Die zwölfte und letzte reguläre Frage des Fragebogens erkundigte sich im Fall einer verneinenden Antwort auf die zehnte Frage danach, warum die Befragten der Meinung sind, dass das Übersetzen von Pressetexten aus Sicht der späteren Übersetzertätigkeit nicht relevant sei. Von den neun Personen, die bei der zehnten Frage so geantwortet hatten, lieferten hier acht auch eine Erklärung für ihre Antwort. Der feststellbare Hauptgrund (fünf von acht Antworten) ist aufgrund der Rückmeldungen die geringe Zahl solcher Aufträge, wobei die diesbezüglichen Formulierungen geringfügig voneinander abweichen (,der Bedarf an Presseübersetzung ist gering“; ,,es gibt keine Übersetzungsaufträge auf diesem Gebiet, lediglich die Pressebeobachtung an Botschaften stellt eine relevante Aufgabe dar“; ,,meiner Erfahrung zufolge erhalten Übersetzer nur selten solche Aufträge / ich zum Beispiel nie/“; , stellt in der beruflichen Praxis der freiberuflichen Übersetzer nur einen kleinen Teil der Aufträge dar“; „während meiner 15-jährigen Laufbahn habe ich höchstens fünf solche Aufträge erhalten“). Zwei Antworten begründeten die fehlende Relevanz damit, dass es sich nur um eine mögliche Textsorte handle („es handelt sich lediglich um eine der möglichen Textsorten“; „Solche Texte weichen von der Übersetzung anderer Texte nicht bedeutend ab. Sie können höchstens wegen der Aktualitäten interessant sein."). Schließlich lautet ein letztes - leider nicht weiter ausgeführtes und damit auch nicht ganz verständliches - Argument so, dass „zwischen den beiden Dingen kein Zusam- 
menhang besteht“" (also zwischen dem Übersetzen von Pressetexten in der Praxis des Übersetzers und dem Befassen mit Pressetexten im Übersetzungsunterricht).

Am Ende des Fragebogens konnten die Befragten freitextliche Anmerkungen zum Fragebogen machen. Zwölf Befragte nutzten diese Möglichkeit, wobei aus Sicht der vorliegenden Studie acht von Bedeutung sind. Sieben Anmerkungen beziehen sich dabei auf folgende Bereiche: die Rolle der Presseübersetzung in der Wissensvermittlung (Allgemeinwissen), bei der Vermittlung aktueller Informationen, der Wortschatzerweiterung, beim Kennenlernen verschiedener Textsorten sowie das Einüben konkreter Übersetzungsstrategien und das Lösen übersetzungstechnischer Probleme. Eine Anmerkung wiederum erwähnt die Rolle der Zweisprachigkeit (Slowakeiungarn) und die Tatsache, dass Redaktionen der Meinung seien, diese Menschen könnten von Haus aus übersetzen. Deshalb stellten sie keine qualifizierten Übersetzer an.

\section{Fazit}

Tatsache ist, dass sich die ungarische translationswissenschaftliche Fachliteratur bislang fast überhaupt nicht bzw. nur aus dem verhältnismäßig engen Aspekt der Textlinguistik mit der journalistischen Translation beschäftigt hat. Die Untersuchung dieser Texte ist jedoch auch im internationalen Vergleich erst recht spät - in den ersten Jahren des 21. Jahrhunderts - in den Fokus der Translationswissenschaft gerückt. Aber auch im Fall der Letzteren wurde meines Wissens bis dato keine ähnliche Studie vorgenommen, die sich auf den Einsatz von Pressetexten im Übersetzungsunterricht konzentriert. Aufgrund der durchgeführten Studie mit 52 Teilnehmern konnte diesen Fakten zum Trotz nachgewiesen werden: Pressetexte spielen in der Übersetzerausbildung in Ungarn und auch in einigen Hochschulinstitutionen dreier Nachbarländer eine grundlegende Rolle, einerseits aufgrund der Verbreitung (derzeit lassen über $85 \%$ der Befragten solche Texte im Unterricht übersetzen), andererseits aufgrund der Durchgängigkeit der Verwendung (fast 50 \% der Befragten lassen während der gesamten Übersetzerausbildung Pressetexte übersetzen). Der Einsatz solcher Texte erfolgt aus einer Vielzahl von Gründen, aus unterschiedlicher Motivation heraus, und es ist nicht ein einziger wichtiger Grund auszumachen: Hierzu gehören zum Beispiel die Vermittlung verschiedener Gattungen/Textsorten, die Erweiterung verschiedener Stile bzw. sprachlicher Register, die Vermittlung übersetzungstechnischer Kenntnisse und die Erweiterung des Wortschatzes.

Die Fragen nach der Relevanz der Presseübersetzung bestätigen und begründen die Präsenz im Übersetzungsunterricht. Das diesbezügliche Ergebnis - ebenfalls über 80 \% der Befragten halten die Presseübersetzung aus Sicht der späteren Arbeit als Übersetzer für relevant - fiel weit deutlicher aus, als ich vorab erwartet habe. Die Befragten sehen die vielfältigen Anwendungsbereiche dieses Teilge- 
bietes der Übersetzung deutlich, wie die entsprechenden Antworten zeigen. Auf der anderen Seite konnte zwar der Grund dafür, weshalb die Übersetzung von Pressetexten aus Sicht der späteren Übersetzertätigkeit der Meinung einiger Dozenten zufolge nicht relevant sei, eindeutig ermittelt werden (zu wenige derartige Aufträge), jedoch das Absehen von der Verwendung im Unterricht aufgrund der gegebenen Antworten nicht eindeutig festgestellt werden. Diesbezügliche Vermutungen habe ich allerdings aufgrund meiner Erfahrung und der freitextlichen Anmerkungen in der vorliegenden Arbeit geäußert.

Die Ergebnisse der durchgeführten Studie bestätigen meines Erachtens die Notwendigkeit der näheren Erforschung der Situation der journalistischen Translation - als eines Teilgebietes der Übersetzungsbranche und der Translationswissenschaft - in Ungarn, der ich mich im Rahmen eines größeren Forschungsprojekts widme. Aufgrund weiterer Untersuchungen können bestimmte Meinungen, Feststellungen von Teilnehmern der hier beschriebenen Studie bestätigt oder widerlegt und es können die eventuellen Zusammenhänge zwischen der Ausbildung von Sprachmittlern und der Durchführung von Übersetzungsaufgaben im Bereich journalistische Translation (vor allem im Kreis von professionellen Journalisten) näher beleuchtet werden.

\section{Literaturverzeichnis}

Baker, Mona - Saldanha, Gabriela (eds.) 2009. Routledge Encyclopedia of Translation Studies. 2nd edition. London-New York: Routledge.

Bani, Sara 2006. An analysis of press translation process. In: Conway, Kyle \& Bassnett, Susan (eds.): Translation in Global News - Proceedings of the conference held at the University of Warwick, 23 June 2006. Coventry: University of Warwick, Centre for Translation and Comparative Cultural Studies, 35-45.

Bánhegyi, Mátyás 2012. Screening Political Bias and Reality in Media Translations. Translation Journal. Online: http://translationjournal.net/journal/61bias.htm. Bánhegyi, Mátyás 2013. Exposing Political Manipulation and Bias in Mediatised Translations. The Translation-centred Political Mass Communication Model. Intralinea.Vol.15. Online:http://www.intralinea.org/archive/article/exposing political_manipulation_and_bias_in_mediatised_translations

Bielsa, Esperanca - Bassnett, Susan 2009. Translation in Global News. LondonNew York: Routledge.

Felekné Csizmazia, Erzsébet 2014. A fordítás és a tolmácsolás határán: a blattolás kutatása. Fordítástudomány. Vol. XVI, No. 2. 24-36.

Gambier, Yves - van Doorslaer, Luc (eds.) 2010. Handbook of Translation Studies. Vol. 1. Amsterdam-Philadelphia: John Benjamins Publishing Company. 
Harris, Brian - Sherwood, Bianca 1978. Translating as an innate skill. In: Gerver, David - Sinaiko, H. Wallace (eds.): Language, Interpretation and Communication. New York-London: Plenum Press, 155-170.

Hu, Gengshen 2003. Translation as adaptation and selection. Perspectives: Studies in Translatology. Vol. 11, No. 4. 283-291.

Károly, Krisztina 2007. Szövegtan és fordítás. Budapest: Akadémiai Kiadó.

Károly, Krisztina et al. 2012. Szövegkohézió és sajtófordítás: Kohéziós eltolódások a hírszövegek magyar-angol fordításában. Fordítástudomány. Vol. XIV, No. 2. 30-67.

Károly, Krisztina 2013. A retorikai struktúra múfaji szempontú elemzésének módszereiről a magyar-angol sajtófordításban. Fordítástudomány. Vol. XV, No. 1. 5-31.

Parkin, Christina 2012. Stehgreifübersetzen. Überlegungen zu einer Grenzform der Translation am Beispiel des Sprachenpaares Französisch-Deutsch. Frankfurt/ Main: Peter Lang Verlag.

Róka, Jolán 1986. Az újságszövegek szerkesztési és stílustipológiája. Budapest: Akadémiai Kiadó.

Valdeón, Roberto A. 2015. Fifteen years of journalistic translation research and more. Perspectives: Studies in Translatology. Special Issue: Culture and news translation. Vol. 23, No. 4. 634-662.

van Doorslaer, Luc 2009. How language and (non-)translation impact on media newsrooms: the case of newspapers in Belgium. Perspectives: Studies in Translatology. Vol. 17, No. 2. 83-92.

Zachar, Viktor 2013a. A neologizmusok fordításának vizsgálata relevanciaelméleti szempontból (német-magyar és magyar-német irányban). PhD-Dissertation. Budapest: Eötvös-Loránd-Universität.

Zachar, Viktor 2013b. Valós fordítási feladatok, valós fordítási problémák - Múfajok és feladattípusok az ELTE Fordító- és Tolmácsképző Tanszék mesterképzésén. Szaknyelv és szakfordítás. 2013. szeptember, 23-30. 